\title{
Role of vacancies in the structural stability of $\alpha$-TiO: A first-principles study based on density-functional calculations
}

\author{
Jesús Graciani, Antonio Márquez, and Javier Fdez. Sanz* \\ Departamento de Química Física, Facultad de Química, Universidad de Sevilla, E-41012 Sevilla, Spain
}

(Received 30 December 2004; revised manuscript received 26 April 2005; published 11 August 2005)

\begin{abstract}
Many transition-metal monoxides formally having an fcc rock-salt structure exhibit a relatively high degree of vacancies, in particular, the most stable structure of stoichiometric titanium monoxide corresponds to a monoclinic phase, $\alpha$-TiO, showing $15 \%$ vacancies. The role of such vacancies on the stability of the solid has been the subject of speculations for the last 30 years. We report in this work a theoretical study of the electronic structure of $\alpha$-TiO based on generalized gradient approximation density functional calculations. Analysis of electron distribution changes induced by the creation of defects on the ideal rock-salt structure allows us to clarify the significant function played by both $\mathrm{O}$ and $\mathrm{Ti}$ ordered vacancies that work together on stabilization of the material. Stabilization of the monoclinic phase with respect to the cubic one involves a noticeable repolarization of the Ti $3 d$ orbitals that simultaneously contribute to two cooperative mechanisms, (i) enhancement of the Ti-Ti bonding interactions through and around the oxygen vacancies, and (ii) electrostatic stabilization resulting from electron density accumulation in a oxygen vacancy (cation environment) together with electron density depletion in titanium vacancy (anionic environment).
\end{abstract}

DOI: 10.1103/PhysRevB.72.054117

PACS number(s): 61.72.Ji, 71.20.Ps

\section{INTRODUCTION}

Titanium monoxide $\mathrm{TiO}$ belongs to a broad family of compounds referred to as hard refractory metals, which includes nitride and carbide binary derivatives of IIIA, IVA, and VA transition metals. These materials exhibit covalent properties (high melting points, extreme hardness) together with metallic conductivity comparable to those of pure transition metals. ${ }^{1}$ They have a rock-salt structure, and a remarkable feature of these monoxides is the occurrence of a high percentage of vacancies in both the oxygen and metal sublattices (up to $25 \%$ in $\mathrm{NbO}$ ). ${ }^{1,2}$

Stoichiometric $\mathrm{TiO}$ spontaneously shows $15 \%$ vacancies in an approximated one to one ratio. After annealing the system at $990{ }^{\circ} \mathrm{C}$ there is an ordering of the vacancies ${ }^{3}$ giving rise to a monoclinic phase known as $\alpha$-TiO with a unit cell of formula $\mathrm{Ti}_{5} \mathrm{O}_{5}$. This behavior indicates that a vacancy ordered structure is thermodynamically favored with respect to the undefective solid. It also makes clear that the vacancy origin is not due to an entropic effect as commonly observed in other solids. ${ }^{4,5}$ Furthermore, keeping pressure and temperature under control it is possible to obtain samples of stoichiometric $\mathrm{TiO}$ within $0-15 \%$ vacancies. ${ }^{6}$ This allows one to analyze the physical properties of the material with respect to the vacancy concentration as reported by Banus and Reed. ${ }^{7}$

How the presence of vacancies in $\mathrm{TiO}$ produces a stabilization of the system has been the subject of contradictory interpretations. The first attempt to provide a qualitative explanation was reported by Goodenough ${ }^{8}$ who proposed that the loss of Madelung energy originated by vacancy formation would be compensated for by an increment of the electron density in the anion holes and by a depletion of the electron density at the cation holes. A second mechanism would involve reinforcement of the metal-metal bond across the vacancy. In 1980, using the non-self-consistent Korringa-
Kohn-Rostoker average $t$-matrix approximation (KKR-ATA), Huisman et al. ${ }^{9}$ estimated the contribution of four main factors, the shift and width of the bands, the shift of the Fermi level, and the appearance of new vacancy states below the Fermi level. Nonempirical Hartree-Fock-Slater calculations reported somewhat later by Gubanov et al. ${ }^{10}$ indicated that the vacancy states were unoccupied and that there was no evidence of a metal-metal bond contribution. However, semiempirical extended Hückel calculations reported by Burdett and Hughbanks ${ }^{11}$ indicated that the metal-metal bond was the main contribution to the stabilization of the monoclinic phase. Further studies agreed on (a) there is an electron density accumulation at the vacancy, (b) there is a reinforcement of the metal-metal bond across the vacancy, and (c) there are occupied vacancy states although their relative energy is not clear. These states have been proposed as falling into the gap between the $\mathrm{O} 2 p$ and $\mathrm{Ti} 3 d$ bands, ${ }^{11,12}$ or in the occupied region of the $\mathrm{Ti} 3 d$ band. ${ }^{13-16}$ In the latest theoretical work addressing this subject, Leung et $a .^{14}$ reported electronic structure calculations using the local density approximation (LDA) of the density functional theory (DFT). These authors concluded that the vacancies allow the valence electrons to delocalize so as to lower the kinetic energy compensating the loss of the Madelung energy.

The electronic structure of stoichiometric $\mathrm{TiO}$ has been experimentally investigated using $\mathrm{x}$-ray photoelectron and emission spectroscopic techniques. ${ }^{17}$ In contrast with band structure calculations, the spectra showed that neither the monoclinic phase nor the cubic samples exhibit a gap. Also, the existence of vacancy states could not be directly verified from experiments, although the narrower valence band of the monoclinic phase with respect to cubic $\mathrm{TiO}$ supported such predictions.

In spite of all this work, it clearly appears that the role played by both oxygen and titanium vacancies still is far from being well understood. Though it seems clear that there 
is some charge accumulation on the oxygen vacancies, the extent has not yet been estimated. Also, contribution of the cation vacancies still remains uncertain. Whether the defects reinforce the strength of a hypothetical $\mathrm{Ti}$ - Ti bond across the vacancy deserves a more careful analysis. Finally, further clarification on the energy position and the nature of surface states is needed. With the aim of getting a deeper insight into these aspects we report in this paper a theoretical study of the structure of $\mathrm{TiO}$ based on density functional calculations performed using the generalized gradient approximation (GGA) to introduce the exchange and electron correlation contributions. The paper is organized as follows: in Sec. II, models and computational methods are described. The results and discussion are presented in Sec. III. Finally, the main conclusions are outlined in Sec. IV.

\section{MODELS AND COMPUTATIONAL DETAILS}

In order to model the extended nature of these surfaces, periodic three-dimensional (3D) DFT calculations were carried out using the VASP 4.6 code $^{18,19}$ and the projector augmented wave method (PAW) ${ }^{20}$ Energy was obtained in these calculations using the GGA implementation of DFT proposed by Perdew et al. ${ }^{21}$ and the valence electronic states were expanded using plane waves as basis set with a cutoff of $500 \mathrm{eV}$. In the case of Ti atoms, the semicore $3 s 3 p$ states were also included. Thermal smearing of one-electron states $\left(k_{B} T=0.05 \mathrm{eV}\right)$ was used, together with the MethfesselPaxton scheme of order two. The number of $k$ points used was selected in order to get $T S$ corrections to the energy less than $0.001 \mathrm{eV}$. They were generated using the MonkhorstPack method, the grids being $8 \times 8 \times 8$ for cubic TiO, and $8 \times 6 \times 8$ for monoclinic TiO.

Forces on the ions were calculated through the HellmannFeyman theorem as the partial derivatives of free energy with respect to the atomic position, including the Harris-Foulkes ${ }^{22}$ correction to forces. This calculation of the forces allows a geometry optimization using the conjugategradient scheme. Iterative relaxation of atomic positions was stopped when the change in total energy between successive steps was less than $0.001 \mathrm{eV}$. With this criterion, forces on the atoms were generally less than $0.1 \mathrm{eV} / \AA$.

Two structures of $\mathrm{TiO}$ have been considered in the computations, cubic and monoclinic. The first is the undefective $\mathrm{NaCl}$ structure and has been used as a vacancy-free reference. The unit cell of the monoclinic phase $\alpha$-TiO is similar to that of $\mathrm{NaCl}$ but has an ordered array of vacant lattice sites. Half of the titanium and half of the oxygen atoms are missing alternately in every third (110) plane. ${ }^{3}$ Since in this phase, with space group $A 2 / m$, there is approximately one formula unit missing in every six units, this stoichiometric compound is usually denoted as $\mathrm{Ti}_{5} \mathrm{O}_{5}$, although the unit cell has 20 atoms. In this structure, Fig. 1, the 3D ordering of vacancies gives rise to octahedral holes of cations and anions aligned along the $\langle 001\rangle$ direction and sharing a vertex. Such an alignment actually results in cation and anion vacancy channels running parallel to the $\langle 001\rangle$ direction as recently observed using high-resolution electronic microscopy (HREM) ${ }^{23,24}$ Concerning the coordination, the atoms lying

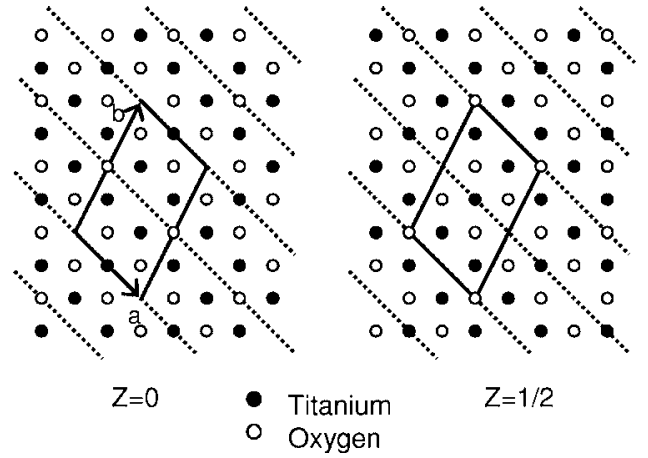

FIG. 1. $z=0$ and $z=1 / 2$ planes of the experimentally resolved $\alpha$-TiO unit cell (Ref. 3). Dashed lines indicate the planes containing vacancies. The monoclinic vectors $\left(a^{\prime}, b^{\prime}, c^{\prime}\right)$ are obtained from the cubic ones $(a, b, c)$ as

$$
\left(\begin{array}{l}
a^{\prime} \\
b^{\prime} \\
c^{\prime}
\end{array}\right)=\left(\begin{array}{ccc}
1 & -1 & 0 \\
1 & 2 & 0 \\
0 & 0 & 1
\end{array}\right)\left(\begin{array}{l}
a \\
b \\
c
\end{array}\right) .
$$

This transforms the first Brillouin zone from a truncated octahedron into a distorted hexagonal prism.

in fully occupied layers, i.e., $80 \%$ of $\mathrm{Ti}$ and $\mathrm{O}$ atoms, are fivefold coordinated in a square pyramidal symmetry, while those in the vacancy containing layers, i.e., the remaining $20 \%$ of the Ti and O atoms, have a square planar fourfold coordination. According to their coordination, there are three different types of $\mathrm{Ti}$ and $\mathrm{O}$ atoms as schematically shown in Fig. 2. Atoms between two vacant sites, belonging to the vacancy channel, are labeled as Ti-CV and O-CV and they are fourfold coordinated. Atoms placed on the vacancy sides are fivefold coordinated and labeled as Ti- $X$ and $\mathrm{O}-X$ if they are aligned with the $X$ axis and Ti- $Y$ and $\mathrm{O}-Y$ if aligned with the $Y$ axis.

\section{RESULTS}

We start this section reporting on the preliminary tests performed to evaluate the reliability of our theoretical approach. With this aim a full relaxation of both the cell parameters and the ion positions has been performed for cubic and monoclinic structures, and the results are reported in Table I. The theoretical lattice parameters estimated here are in agreement with the experimental data for both structures,

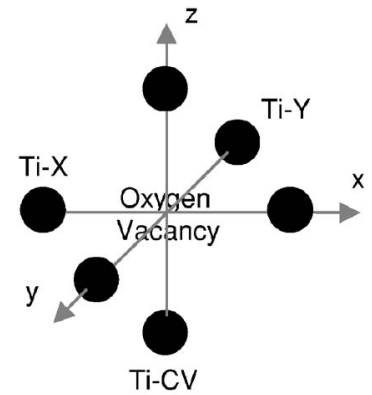

(a)

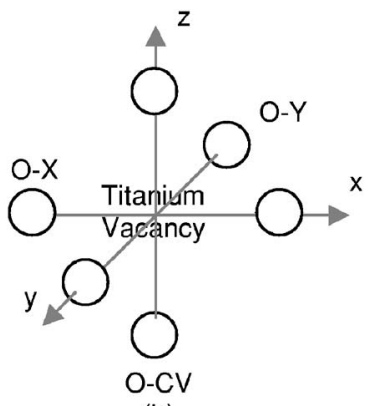

(b)
FIG. 2. Schematic representation of (a) O vacancy and (b) Ti vacancy. 
TABLE I. Theoretical and experimental lattice parameters and heats of formation of stoichiometric titanium oxide. Cubic, rock-salt vacancy-free structure; $\alpha$-TiO monoclinic phase.

\begin{tabular}{|c|c|c|c|}
\hline & $\begin{array}{c}\text { Present } \\
\text { work GGA }\end{array}$ & $\mathrm{LDA}^{\mathrm{a}}$ & Experiment ${ }^{b}$ \\
\hline \multicolumn{4}{|l|}{ Cubic } \\
\hline$a / \AA$ & 4.252 & & 4.204 \\
\hline$\Delta \mathrm{H} / \mathrm{kJ} \mathrm{mol}^{-1}$ & -462.4 & & \\
\hline \multicolumn{4}{|l|}{ Monoclinic } \\
\hline$a / \AA$ & 5.808 & 5.730 & 5.855 \\
\hline$b / \AA$ & 9.283 & 9.278 & 9.340 \\
\hline$c / \AA$ & 4.142 & 4.136 & 4.142 \\
\hline$\gamma$ & $108^{\circ}$ & $107^{\circ}$ & $107^{\circ} 32^{\prime}$ \\
\hline$\Delta \mathrm{H} / \mathrm{kJ} \mathrm{mol}^{-1}$ & -507.6 & -648.4 & -519.7 \\
\hline
\end{tabular}

${ }^{\mathrm{a}}$ Reference 14.

${ }^{\mathrm{b}}$ References 3 and 25 .

with errors lesser than $0.8 \%$ for $\alpha$-TiO and $1.15 \%$ for cubic phase. Compared with the LDA calculations reported by Leung et al. ${ }^{14}$ it turns out that the GGA computations give superior agreement. We have also estimated the heat of formation of both structures (Table I), computed as the difference between total energies of titanium oxide and the sum of crystalline titanium and molecular oxygen. For the monoclinic structure the heat of formation is computed to be $-507.6 \mathrm{~kJ} / \mathrm{mol}$, in excellent agreement with the experimental value, $-519.7 \mathrm{~kJ} / \mathrm{mol}^{25}$ This is at variance with the LDA predicted value of $-648.4 \mathrm{~kJ} / \mathrm{mol}$ that clearly appears to be overestimated. On the other hand, the cubic phase is less stable than the monoclinic structure, in agreement with previous work, the difference predicted by our GGA calculations being $45.2 \mathrm{~kJ} / \mathrm{mol}$, indicating that vacancy formation stabilizes the structure.

\section{A. Electronic structure}

The general effect that vacancies formation has on the electronic structure can be traced from the total density of states, DOS, curves displayed in Fig. 3. Taking cubic TiO as reference (bottom of the figure), vacancy creation induces three main features. First, Ti $3 s$ and Ti $3 p$ bands split showing new components at higher energies. Also a shoulder in the $\mathrm{O} 2 s$ band is observed. Second, there is a general shift of about $0.6 \mathrm{eV}$ towards higher energy. Finally, the gap between the $\mathrm{O} 2 p$ and $\mathrm{Ti} 3 d$ bands lowers by $0.54 \mathrm{eV}$.

To rationalize these effects we will consider in more detail the band components evolution, reported separately in Figs. 4 (semicore states) and 5 (valence states) for the sake of the analysis. Starting from the cubic phase, creation of a defect leads to a configuration in which six ions with the same charge are facing each other, with a strong increment of electrostatic repulsion [see Figs. 2(a) and 2(b)]. This repulsion induces a destabilization of the semicore bands that shift to higher energies. Such a shift should be larger for those bands arising from atomic orbitals pointing towards the vacancy. Indeed, a higher destabilization is expected in the bands as-

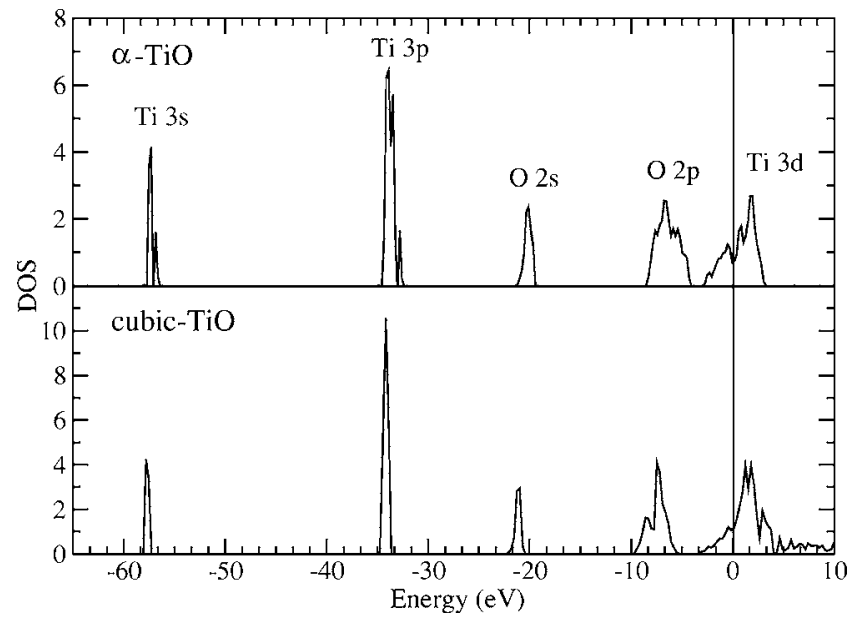

FIG. 3. Total density of states (per TiO unit) for $\alpha$-TiO and cubic-TiO structures. The vertical line at $0 \mathrm{eV}$ shows the Fermi level.

sociated with the vacancy channels Ti-CV and O-CV atomic sites as these ions feel the repulsion of 10 ions of the same charge. In contrast, those ions on the $X$ and $Y$ sites only feel the repulsion of five ions with the same charge. This anisotropy gives rise to different polarization of the electron clouds of $X / Y$ and $\mathrm{CV}$ ions. This is certainly the qualitative behavior observed in Fig. 4. All semicore bands shift, however, the destabilization of Ti- $X 3 p_{x}$ and Ti- $Y 3 p_{y}$ and, mainly, Ti-CV $3 p_{z}$ components are larger. We can now see how the splitting observed in the total DOS of Fig. 3 is due to the differential shift of the $3 s$ and $3 p_{z}$ states of titanium due to the anisotropy of the structure.

Focusing now on the valence bands, qualitative similar features should occur, although larger effects are expected because of their higher polarizable nature. As shown in Fig.
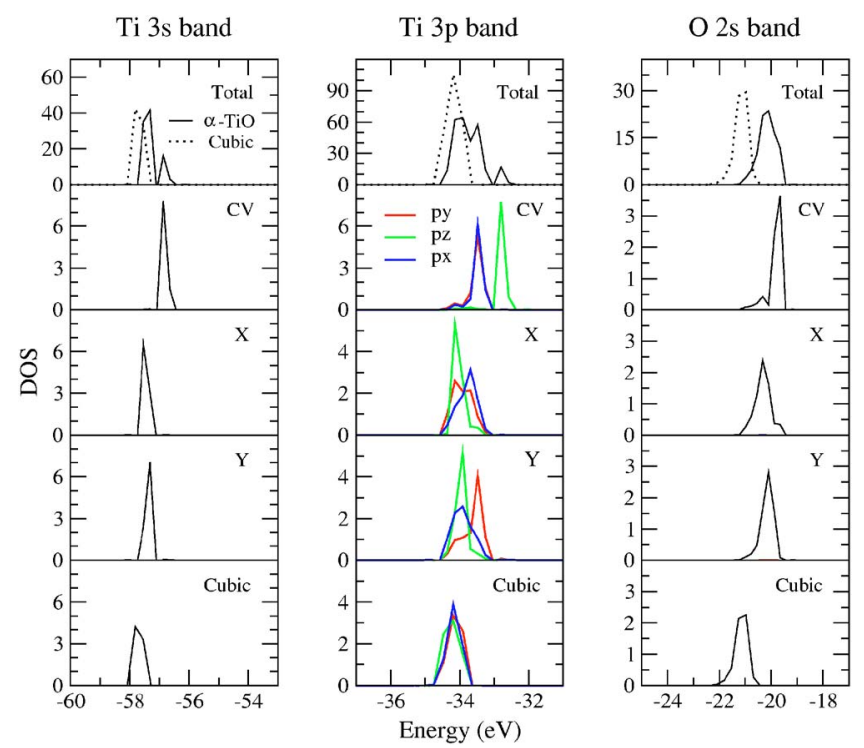

FIG. 4. (Color online) Total density of states for $\alpha$-TiO and cubic-TiO structures, and projected density of states on $\mathrm{Ti}$ and $\mathrm{O}$ ions at the $\mathrm{CV}, X$ and $Y$ positions as well as cubic phase for the indicated bands. 
O 2p band

Ti 3d band

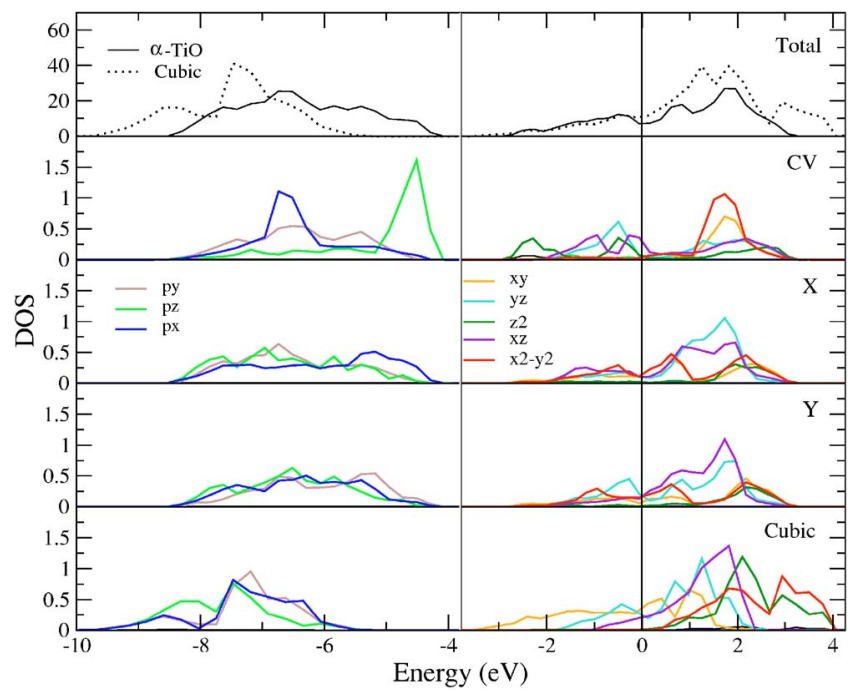

FIG. 5. (Color online) Total density of states for $\alpha$-TiO and cubic-TiO structure, and projected density of states on $\mathrm{Ti}$ and $\mathrm{O}$ ions at the $\mathrm{CV}, X$ and $Y$ positions, and cubic phase for the indicated bands. The vertical line at $0 \mathrm{eV}$ shows the Fermi level.

5, O-X $2 p_{x}$ and O-Y $2 p_{y}$ states shift significantly though the main feature is the huge shift observed for the $2 p_{z}$ states originated by the O-CV vacancy channel atoms. The evolution of Ti $3 d$ states is more involved as can be seen in Fig. 5. There are not only band shifts, but some bands that were below the Fermi level fall now above it, and vice versa. Before going on to the analysis, a further point concerning the $\mathrm{O} 2 p-\mathrm{Ti} 3 d$ band gap deserves our attention. Indeed, the fact that this band gap is relatively large, $\sim 2 \mathrm{eV}$, indicates that the $\mathrm{O} 2 p$ and Ti $3 d$ hybridation is low and therefore the $3 d$ band is mainly built up from the Ti-Ti interaction, i.e., metal-metal bond. The changes induced on these bands are then reflecting the changes of the Ti-Ti bonding. As can be observed in Fig. 5, the Ti-CV $3 d_{x y}$ component shifts above the Fermi level while the Ti-CV $3 d_{z^{2}}$ states now are found below it. The gain/loss of charge for a given component may be roughly estimated by integrating the bands up to the Fermi level. Thus, the $75 \%$ of the electron density lost by the Ti $3 d_{x y}$ orbitals goes to the Ti-CV $3 d_{z^{2}}$ states. The remaining $25 \%$ increases the population of the Ti-CV $3 d_{x z}, 3 d_{y z}$ orbitals. These changes make clear the strong repolarization of the Ti-CV centers when two oxygen atoms are removed. The Ti electronic clouds are strongly attracted by the surrounding cations, and spread towards the vacancy. The large stabilization of the Ti-CV $3 d_{z^{2}}$ states, induced by cation coordination, clearly indicates a reinforcement of the metal bond between Ti-CV atoms across the vacancy. The stabilization of Ti-CV $3 d_{x z}, 3 d_{y z}$ states also indicate a larger bonding with Ti- $X$ and Ti- $Y$, but to a lesser extent. It is worth noting on the other hand that both the destabilization of the $\mathrm{O} 2 p_{z}$ and the stabilization of the Ti $3 d_{z^{2}}$ states are responsible for lowering the $p-d$ band gap referred to above.

A similar behavior is observed for Ti- $X$ and Ti- $Y$ atoms, but now the components stabilized by the vacancy (those pointing towards it) are the $3 d_{x^{2}-y^{2}}$, now below the Fermi (a)

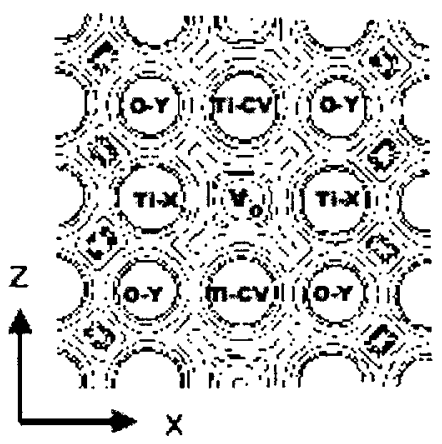

(b)

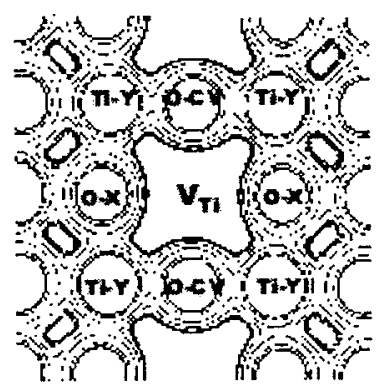

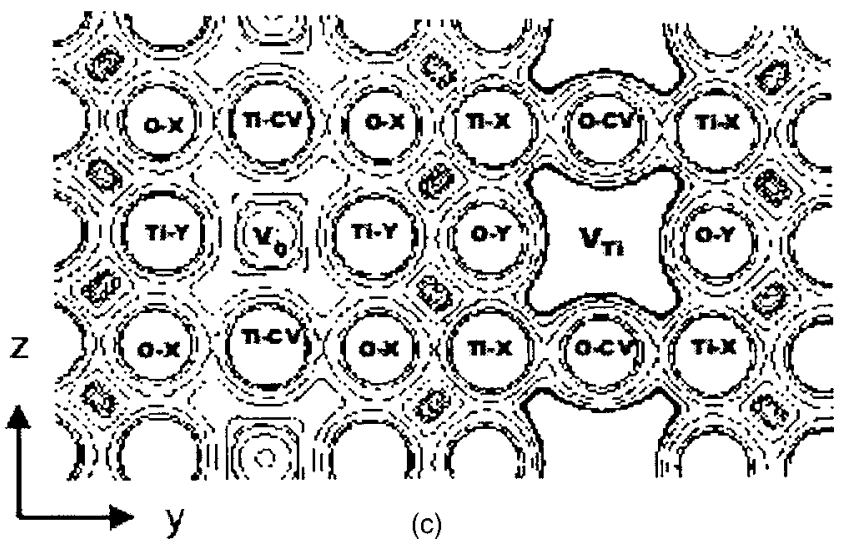

FIG. 6. Electron density maps, (a) $X Z$ plane containing Ti- $X$ ions ( $3 d_{x z}$ band), (b) $X Z$ plane containing Ti- $Y$ ions ( $3 d_{x z}$ band), and (c) $Y Z$ plane containing all types of $\mathrm{Ti}$ ions ( $3 d_{y z}$ band).

level, although, as expected, their shifts are not as large as those of Ti-CV $3 d_{z^{2}}$ states. The $3 d_{x y}$ bands in their turn are strongly destabilized with a significant loss of electron population. Another interesting feature is the repolarization of the $3 d_{x z}$ and $3 d_{y z}$ bands. In the case of Ti- $X$, the $3 d_{x z}$ band gains electron population at the expense of the $3 d_{y z}$ one, with a $3 d_{x z} / 3 d_{y z}$ ratio of 1.5. This rehybridation allows for a better overlap between $\mathrm{Ti}$ orbitals reinforcing the bond between $\mathrm{Ti}-X$ and Ti-CV atoms. In the case of Ti- $Y$ centers, the repolarization goes in the inverse sense, the $3 d_{y z}$ population increases and the $3 d_{x z}$ diminishes, with a $3 d_{y z} / 3 d_{x z}$ ratio of 2.5 , with an improvement of the bond with Ti-CV atoms.

\section{B. Electron density maps}

Further insight into the electronic changes induced by the vacancies may be obtained from analysis of the electron density maps. In Fig. 6, some of these maps corresponding to selected planes are reported. Figures 6(a) and 6(b) are planes parallel to the $X Z$ plane and shows the $3 d_{x z}$ component of the Ti $3 d$ band. Figure 6(a) corresponds to the plane containing $\mathrm{Ti}-X$ and Ti-CV atoms, as well as the vacancy oxygen sites. One can observe that the electron density does not vanish inside the vacancy, and in fact there is some accumulation of charge in the hole. The electron density between $\mathrm{Ti}$ atoms reaches a minimum at the middle point of an imaginary line connecting them, and then increases back to the Ti centers. This electron pattern is in agreement with the presence of 

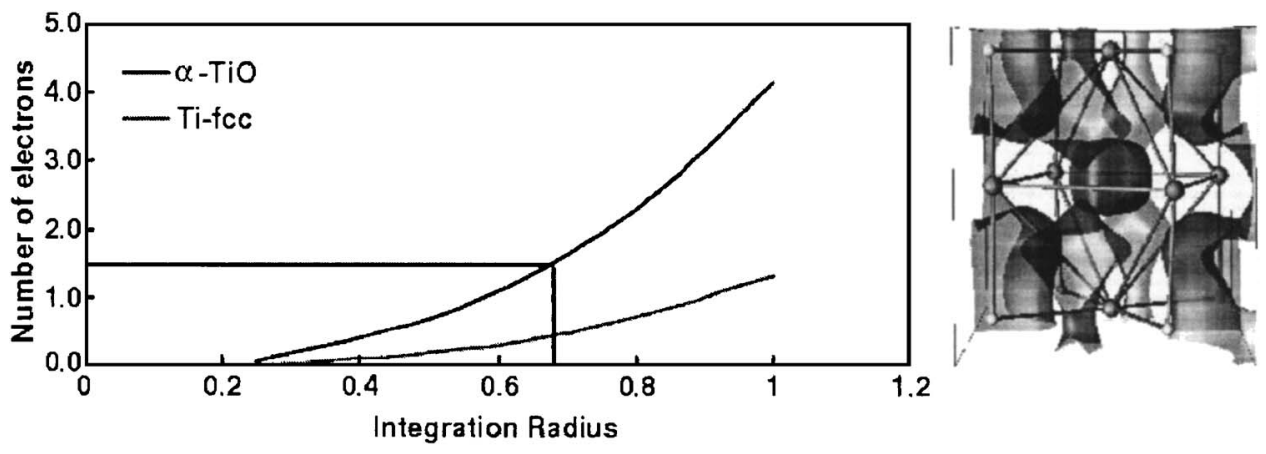

FIG. 7. (a) Electron density integrated in spheres of increasing radii $(\AA)$ centered at the oxygen vacancy. The vertical line shows the radius value for the limit isodensity. (b) Three-dimensional representation of the limit isodensity at an oxygen vacancy. some Ti-Ti metal bond. In Fig. 6(b), we have the Ti vacancies and the Ti- $Y$ atoms. Compared to the Fig. 6(a) map, the most outstanding feature is that the vacancy is completely empty. The $3 d_{x z}$ orbitals pointing towards the hole are strongly repelled by the six anions surrounding them and consequently there is a contraction. This repulsion produces destabilization of the levels, and it is expected that such a destabilization will increase with the number of Ti vacancies, which in this plane amounts to $50 \%$. Figure 6(c) shows the electron density map in the $Y Z$ plane. This plane has three kinds of Ti atoms, with the $3 d_{y z}$ component, and two kinds of vacancies, with a concentration of $\mathrm{Ti}$ vacancies of $25 \%$. We can see again how the $\mathrm{O}$ vacancies accumulate charge while the $\mathrm{Ti}$ vacancies are density free regions. This distribution electrostatically stabilizes the system since actually it involves alternation of vacancies negatively and positively charged, thus partially recovering the Madelung potential. A further aspect of the electrostatic stabilization comes from the fact that there is an enhancement of negative charge in a cation coordination environment, and vice versa, there is an increment of positive charge in a negative environment.

As already mentioned, the stabilization of the Ti $3 d$ components depends on the concentration of Ti vacancies on the same plane. The Ti- $X 3 d_{x z}$ component [Fig. 6(a)] has no Ti vacancies; however, the Ti-X $3 d_{y z}$ component [Fig. 6(c)] finds $25 \%$ vacancies repelling its electron density. This fact explains the observed repolarization for this atom, for which the ratio $3 d_{x z} / 3 d_{y z}$ was 1.5 . In the case of $\mathrm{Ti}-Y$ atoms, the $3 d_{y z}$ component feels $25 \% \mathrm{Ti}$ vacancies, while the concentration of vacancies for the $3 d_{x z}$ component is $50 \%$, consequently the ratio $3 d_{y z} / 3 d_{x z}$ must be larger (estimated to be 2.5).

An interesting point concerning the electron density accumulation in the oxygen vacancies refers to the role that it plays on the final stabilization of the system. Some authors suggested that this charge is a consequence of the Ti-Ti bond through the vacancy, ${ }^{10,11,14}$ while in other works it has been proposed that the charge accumulation is mainly due to electrostatic effects. ${ }^{8,9}$ In order to estimate the weight of these two contributions we have performed a computation of a system identical to $\alpha$-TiO but without the oxygen atoms. This system consists of unoxidized $\mathrm{Ti}$ atoms and therefore the accumulation electron density in the vacancies, if any, must be due to $\mathrm{Ti}$ - Ti metal bonds across the hole since no electrostatic interactions are present. To estimate the charge accumulation we have integrated the electron density in a sphere centered at the vacancy and the results are reported in
Fig. 7(a) for both $\alpha$-TiO and Ti-fcc structures. As can be seen, there is some charge in the Ti-fcc system holes, which reflects the metal-metal bond across the vacancy from overlap of $3 d_{z^{2}}$ and $3 d_{x^{2}-y^{2}}$ orbitals. However, when the oxygen atoms are introduced in the structure the charge accumulation is significantly enhanced (by a factor of about 3), clearly indicating the decisive role of the electrostatic interaction on such an accumulation. In an approximate way, the metalmetal bond accounts for $1 / 3$ of the charge, and the electrostatic interaction for the remaining $2 / 3$. This result agrees with the ionic character observed in the previous theoretical and experimental works. ${ }^{26-29}$ To end this analysis we could estimate the size of the charge accumulated in the vacancies by integrating the electron density in a sphere representing the defect. The radius of the sphere that we have chosen corresponds to that of the electron density isoline, which closes around the vacancy instead of around the Ti neighbors [Fig. 7(b)]. The integration gives a negative charge of $-1.65 e$ and therefore there is an almost complete recovering of the formal charge that would be assigned to an oxygen atom in the defect-free structure. In other words, the Madelung energy is practically recovered by the charge accumulation.

\section{STABILIZATION MECHANISM AND CONCLUDING REMARKS}

The analysis reported in this work, based in GGA calculations, has shown that upon oxygen vacancy creation there is a strong repolarization of the electronic cloud around the metal ions along two main directions (i) reinforcement of the metal-metal bond across the vacancy through $\left(3 d_{x y}\right)$ $\rightarrow\left(3 d_{z^{2}}, 3 d_{x^{2}-y^{2}}\right)$, and around the vacancy through $3 d_{x z} \leftrightarrow 3 d_{y z}$ repolarizations, and (ii) charge accumulation in the vacancy mainly through $\left(3 d_{x y}\right) \rightarrow\left(3 d_{z^{2}}, 3 d_{x^{2}-y^{2}}\right)$ electron pumping which increases the electron density in a cation coordination environment, with the subsequent electrostatic stabilization. Creation of $\mathrm{Ti}$ vacancies in the system also leads to significant changes in the electron distribution as it induces repolarization of the $3 d_{x z}, 3 d_{y z}$ orbitals in order to decrease the electron density in an anionic coordination environment. These changes also favor the metal-metal bond around an oxygen vacancy, the $3 d_{x z}$ between $\mathrm{Ti}-\mathrm{CV}$ and $\mathrm{Ti}$ $-X$; the $3 d_{y z}$ between Ti-CV and Ti- $Y$.

The charge redistribution observed in the $\alpha$-TiO structure obeys the anisotropy introduced by the vacancy channels along the $\langle 001\rangle$ direction. The particular relative disposition of these channels allows larger stabilization of the crystal as 
they produce a reinforcement of the $\mathrm{Ti}-\mathrm{Ti}$ metal bonding across the vacancy. Moreover, for that given vacancy concentration in stoichiometric $\mathrm{TiO}$, the relative disposition of both cation and anion vacancies synergistically acts on the system stabilization. It is also worth noting that such a vacancy ordering actually accounts for the monoclinic structure of $\alpha$-TiO.

If we compare cubic and monoclinic structures we see that the differences rely not only on the vacancies but also in the atomic relaxations. Then, one can wonder if the larger stability of the $\alpha$-TiO structure $(45.2 \mathrm{~kJ} / \mathrm{mol})$ is exclusively due to the polarization arising from the anisotropy introduced by the holes. A simple way to estimate this contribution is to perform a calculation of $\mathrm{TiO}$ structure in which the atoms preserve the cubic defect free positions but with the vacancies corresponding to the monoclinic ordering. Our calculations show that this structure is more stable than the cubic one by $34.3 \mathrm{~kJ} / \mathrm{mol}$ ( $76 \%$ of the difference). The analysis of the DOS reveals that there is a strong repolarization of the $3 d$ band, decreasing electronic density from the anion cavities and placing it into the cation environment. If this structure is allowed to relax, the $\mathrm{Ti}-\mathrm{Ti}$ distance across the vacancy channels diminish while the $\mathrm{O}-\mathrm{O}$ distances increase, recovering the remaining $10.9 \mathrm{~kJ} / \mathrm{mol}$. This analysis makes clear that the charge redistribution constitutes the main contribution to the stabilization mechanism of the $\alpha$-TiO structure via vacancy. These results confirm the stabilization mechanism proposed by Goodenough in its pioneering qualitative work.

This mechanism may also be working for other solids featuring vacancies provided that three requirements are accomplished. First, the material must be ionic enough in order to achieve high electrostatic stabilization. Second, to have an efficient metal-metal bonding contribution, the size of $3 d$ metallic orbitals must be suitable to favor their overlap. Third, the electron configuration of the transition metal atom must be appropriate in order to establish the metal-metal bond. These requirements are fulfilled for instance for the earlier transition metals oxides, $\mathrm{TiO}, \mathrm{ZrO}, \mathrm{VO}, \mathrm{NbO}$. Indeed, the experimental data indicate that $\mathrm{VO}$ and $\mathrm{NbO}$ show $15 \%$ and $25 \%$ vacancies in both sublattices.

\section{ACKNOWLEDGMENTS}

Financial support from the Spanish Ministerio de Ciencia y Tecnologia (MAT2002-0576), and the Junta de Andalucía (FQM-132), is appreciated.
*Corresponding author. Electronic address: sanz@us.es

${ }^{1}$ S. P. Denker, J. Less-Common Met. 14, 1 (1967).

${ }^{2}$ A. F. Wells, Structural Inorganic Chemistry (Clarendon, Oxford, England, 1975).

${ }^{3}$ D. Watanabe, J. R. Castles, A. Jostsons, and A. S. Malin, Acta Crystallogr. 23, 307 (1967).

${ }^{4}$ N. W. Ashcroft and N. D. Mermin, Solid State Physics (Holt, Rinehart, and Winston, New York, 1976), Chap. 30, p. 616.

${ }^{5}$ C. Kittel, Introduction to Solid State Physics (Wiley, New York, 1986).

${ }^{6}$ A. Taylor and N. J. Doyle, High Temp. - High Press. 1, 679 (1969).

${ }^{7}$ M. D. Banus, T. B. Reed, and A. J. Strauss, Phys. Rev. B 5, 2775 (1972).

${ }^{8}$ J. B. Goodenough, Phys. Rev. B 5, 2764 (1972).

${ }^{9}$ L. M. Huisman, A. E. Carlsson, C. D. Gelatt, and H. Ehrenreich, Phys. Rev. B 22, 991 (1980).

${ }^{10}$ V. A. Gubanov, A. L. Ivanovsky, G. P. Shveikin, and D. E. Ellis, J. Phys. Chem. Solids 45, 719 (1984).

${ }^{11}$ J. K. Burdett and T. Hughbanks, J. Am. Chem. Soc. 106, 3101 (1984).

${ }^{12}$ G. Hobiger, P. Herzig, R. Eibler, F. Schlapanski, and A. Neckel, J. Phys.: Condens. Matter 2, 4595 (1990).

${ }^{13}$ S. R. Barman and D. D. Sarma, Phys. Rev. B 49, 16141 (1994).

${ }^{14}$ C. Leung, M. Weinert, P. B. Allen, and R. M. Wentzcovitch, Phys. Rev. B 54, 7857 (1996).

${ }^{15}$ G. Hörmandinger, J. Redinger, P. Weinberger, G. Hobiger, and P. Herzig, Solid State Commun. 68, 467 (1988).
${ }^{16}$ F. Schlapanski, P. Herzig, R. Eibler, G. Hobiger, and A. Neckel, Z. Phys. B: Condens. Matter 75, 187 (1989).

${ }^{17}$ S. Bartkowski, M. Neumann, E. Z. Kurmaev, V. V. Fedorenko, S. N. Shamin, V. M. Cherkashenko, S. N. Nemnonov, A. Winiarski, and D. C. Rubie, Phys. Rev. B 56, 10656 (1997).

${ }^{18}$ G. Kresse and J. Hafner, Phys. Rev. B 47, R558 (1993).

${ }^{19}$ G. Kresse and J. Furthmuller, Comput. Mater. Sci. 6, 15 (1996); Phys. Rev. B 54, 11169 (1996).

${ }^{20}$ G. Kresse and D. Joubert, Phys. Rev. B 59, 1758 (1999).

${ }^{21}$ J. P. Perdew, J. A. Chevary, S. H. Vosko, K. A. Jackson, M. R. Pederson, D. J. Singh, and C. Fiolhais, Phys. Rev. B 46, 6671 (1992).

${ }^{22}$ J. Harris, Phys. Rev. B 31, 1770 (1985); W. M. Foulkes and R. Haydock, ibid. 39, 12520 (1989).

${ }^{23}$ A. A. Valeeva, G. Tang, A. I. Gusev, and A. A. Rempel, JETP Lett. 77, 25 (2003).

${ }^{24}$ A. A. Valeeva, G. Tang, A. I. Gusev, and A. A. Rempel, Phys. Solid State 45, 87 (2003).

${ }^{25}$ Handbook of Chemistry and Physics, 85th ed. (Chemical Rubber Company, Cleveland, 2004), pp. 5-23.

${ }^{26}$ A. A. Valeeva, A. A. Rempel, W. Sprengel, and H. E. Shaefer, Phys. Chem. Chem. Phys. 5, 2304 (2003).

${ }^{27}$ J. K. Burdett, Inorg. Chem. 24, 2244 (1985).

${ }^{28}$ A. Fahmi, C. Minot, B. Silvi, and M. Causá, Phys. Rev. B 47, 11717 (1993).

${ }^{29}$ R. Hengerer, B. Bolliger, M. Erbudak, and M. Grätzel, Surf. Sci. 460, 162 (2000). 